\title{
Climate Change in the European Alps
}

The European Alps are home to diverse ecosystems due to their complex orography and their position between the temperate European climate and the Mediterranean climate. At the same time, the Alps are densely populated and intensively used for tourism, transportation, hydropower, agricultural activities and industrial purposes. The famous glaciers of the Alps are not only a touristic asset, but also a water resource and a key part of Alpine countries' identity.
Stefan Brönnimann

Marcos Andrade Henry F. Diaz

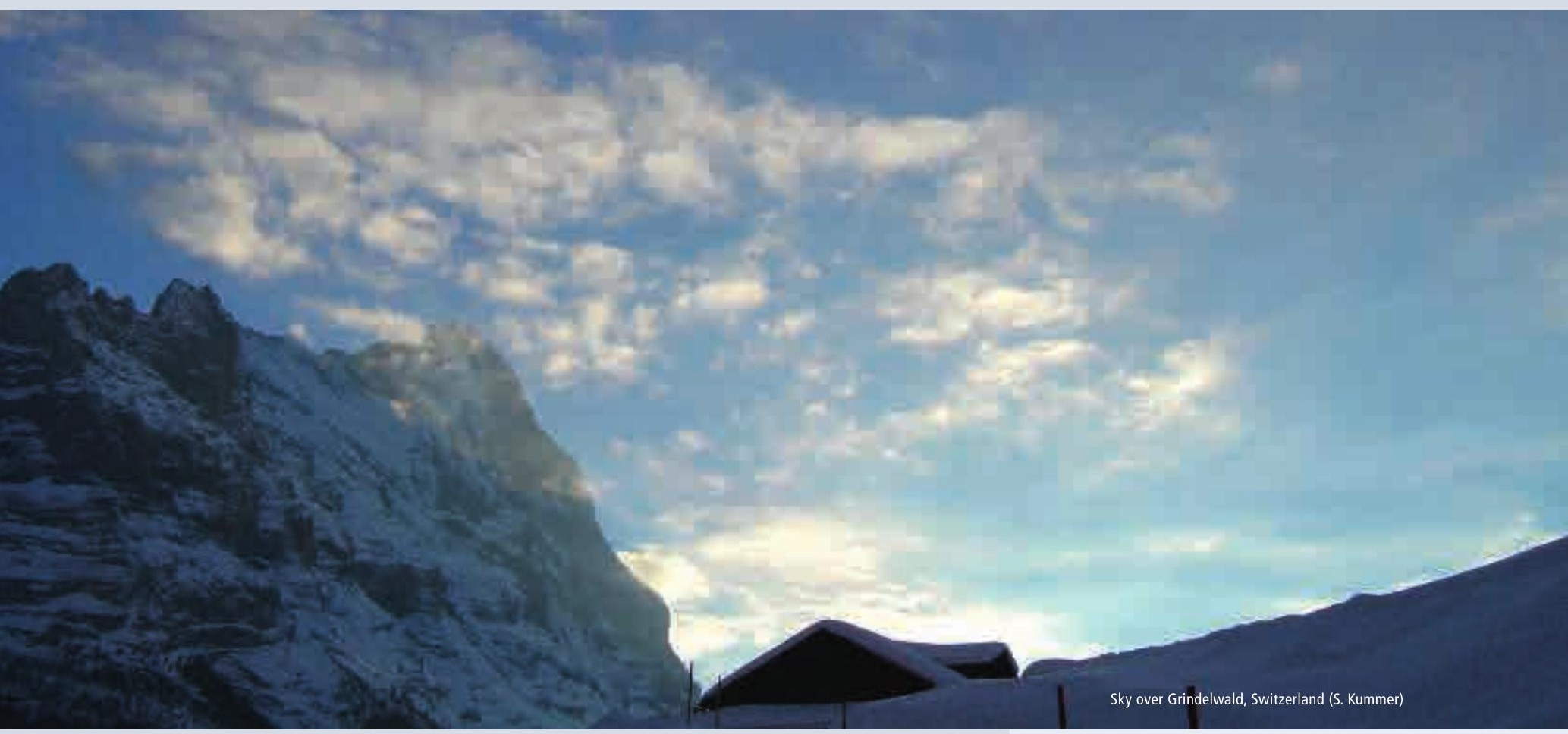

Climate indicators are well monitored in the European Alps, and many data series reach back into the nineteenth century. Temperatures have increased by about $0.12-0.20{ }^{\circ} \mathrm{C}$ per decade over the last 100 years, with a particularly pronounced increase since the mid-1980s [1]. This recent Alpine warming occurred at about three times the rate of the global average. The regional warming trend exhibits little dependence on altitude. In fact, temperatures at altitudes above $4000 \mathrm{~m}$ were found to increase at the same rate as in the lowlands [2]. In addition to mean temperatures, temperature extremes have also changed. In particular, the frequency of warm days has increased and the frequency of cold nights has decreased [3].

Regional precipitation changes were small over the last century, and the main factor affecting snowpack was temperature. At altitudes around $700 \mathrm{~m}$, winter snow is particularly sensitive to temperature changes [4]. Studies show that Alpine snowpack has decreased since the mid-1980s - with high year-to-year variability - and that the snow season has shortened [5].

Summer temperatures are an important variable affecting the balance of glacier mass. The zero-degree level in summer has risen about $75 \mathrm{~m}$ per decade since 1959 [6]. Alpine glaciers have been receding since the 1980s. In terms of mass, the current loss rate for a sample of eight Alpine glaciers is estimated to be 2-3 percent per year

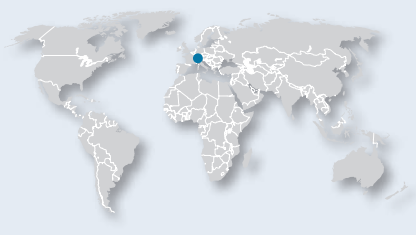




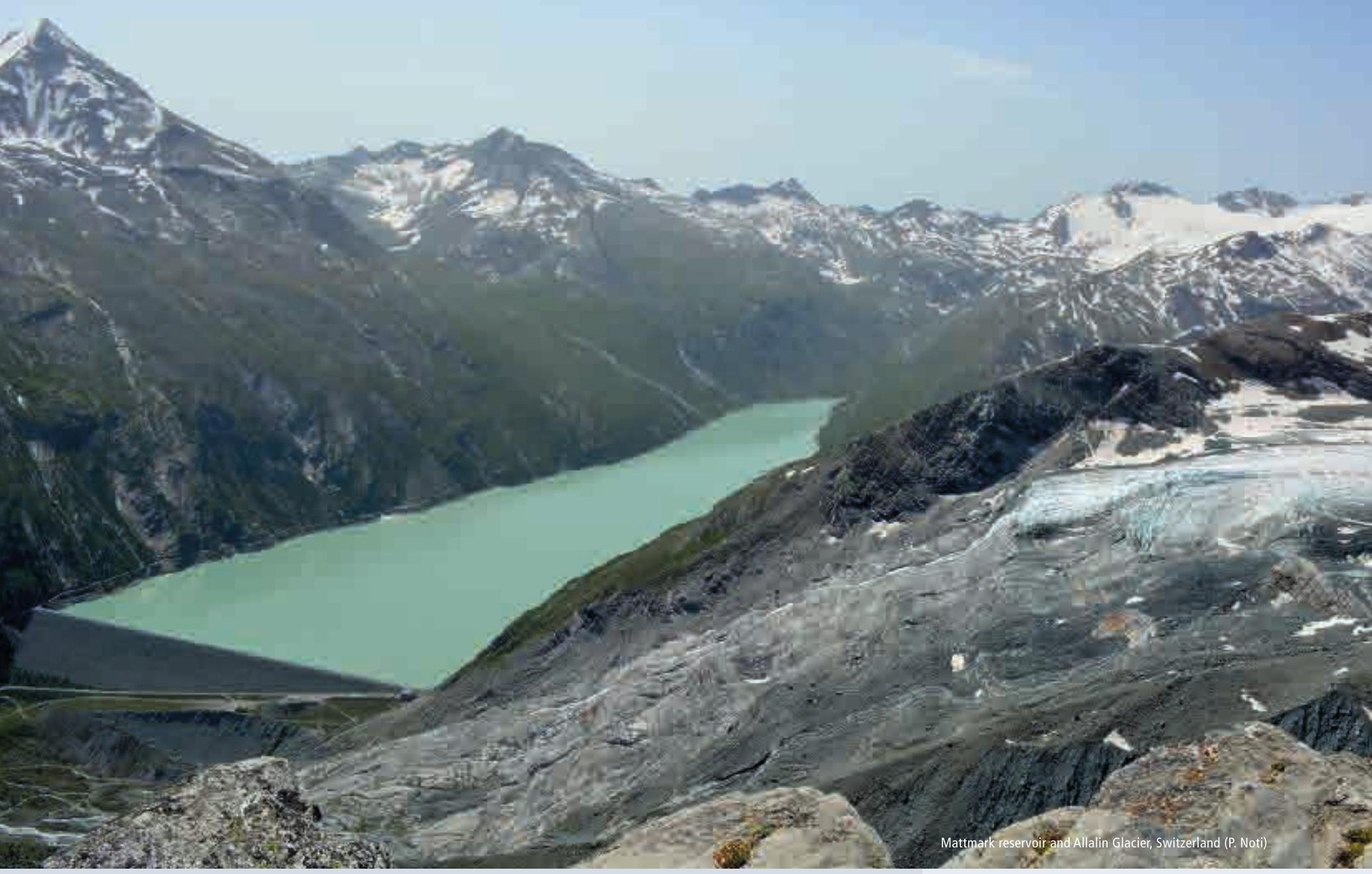

[7]. The temperature changes also affect vegetation. Shifts in vegetation distribution have already been observed in Switzerland, such as an increase in lowland forest species at mid-elevations and an upward shift in the occurrence of certain species [8].

Studies on future climate change scenarios in Switzerland point to acceleration in year-round warming and reductions in summer precipitation [9]; analyses produced for Austria suggest the same [10]. Climate change impacts in Alpine regions are expected to continue unabated or to intensify. Indeed, glaciers in Austria [11] and Switzerland will continue to retreat in the twenty-first century. A recent Climate Change Impact Assessment [12] for Switzerland projected a near-complete loss of glacial ice by the end of the century under a medium-to-high emissions scenario (A1B). Runoff regimes are projected to change from snow-controlled to rain-controlled. Tree species diversity and biomass in high-elevation forests are projected to increase, whereas forests in dry inner-Alpine valleys may deteriorate even under moderate warming [12]. 